\title{
Evaluación electromiográfica del nervio facial y de nervios donadores previo a cirugía de reanimación facial en pacientes con síndrome de Moebius
}

\author{
Electromyographic evaluation of the facial and motor donor nerves before facial \\ reanimation surgery in Moebius syndrome
}

\section{José E. Telich-Tarriba ${ }^{1,2 *}$ y Alexander Cárdenas-Mejía ${ }^{2,3}$}

${ }^{1}$ Servicio de Cirugía Plástica y Reconstructiva, División de Cirugía Plástica y Reconstructiva, Hospital General Dr. Manuel Gea González; ${ }^{2}$ División de Estudios de Posgrado, Universidad Nacional Autónoma de México; ${ }^{3}$ Servicio de Clínica de Nervio Periférico, División de Cirugía Plástica y Reconstructiva, Hospital General Dr. Manuel Gea González. Ciudad de México, México

\section{Resumen}

Antecedentes: El síndrome de Moebius es una entidad clínica caracterizada por parálisis bilateral congénita del nervio facial y el abducens. Adicionalmente se pueden encontrar afectados otros pares craneales. Actualmente no existen estudios que reporten la respuesta electromiográfica de la musculatura facial en esta población. Objetivo: Describir la respuesta electromiográfica de la musculatura facial y de los principales nervios donadores para reconstrucción facial dinámica en pacientes con síndrome de Moebius. Método: Se analizaron electromiografías de los nervios facial, hipogloso, maseterino (trigémino) y accesorio en pacientes con síndrome de Moebius atendidos entre 2010 y 2016. Los resultados se presentan en porcentajes y medidas de tendencia central y de dispersión. Resultados: Se incluyeron 24 pacientes, con una edad promedio de $11.79 \pm 9.39$ años. El nervio facial presentó reclutamiento unilateral completo en 4 pacientes, parcial bilateral en 11 pacientes, en 7 pacientes ausencia de reclutamiento bilateral e inactividad unilateral en 2 pacientes. El maseterino tuvo reclutamiento completo en 14 pacientes, parcial bilateral en 4 pacientes y parcial unilateral en 4 pacientes. El nervio accesorio mostró reclutamiento completo en 20 pacientes, parcial bilateral en 3 pacientes y parcial unilateral en 1 paciente. El hipogloso mostró actividad normal en 22 pacientes, parcial bilateral en 1 paciente y parcial unilateral en 1 paciente. Conclusión: Los pacientes con síndrome de Moebius presentan alteraciones en los registros electromiográficos del nervio facial. La electromiografía permite identificar los nervios donadores para realizar la reconstrucción dinámica.

Palabras Clave: Electromiografía. Nervio facial. Neurofisiología. Parálisis facial. Síndrome de Moebius.

\begin{abstract}
Background: Moebius syndrome is a clinical entity characterized by bilateral facial and abducens nerve palsies; other cranial nerver might be affected as well. So far, no studies have reported the electromyographic responses of the facial musculature in this group of patients. Objective: The objective of our study is to describe the electromyographic responses of the facial muscle and the main donor nerves for facial reanimation in patients with Moebius syndrome. Method: We analyzed electromyographies from the facial, hypoglossal, masseterine (trigeminal) and accessory nerves from patients with Moebius syndrome treated between 2010 and 2016. Results are presented as percentages and central tendency measures. Results: 24 patients
\end{abstract}

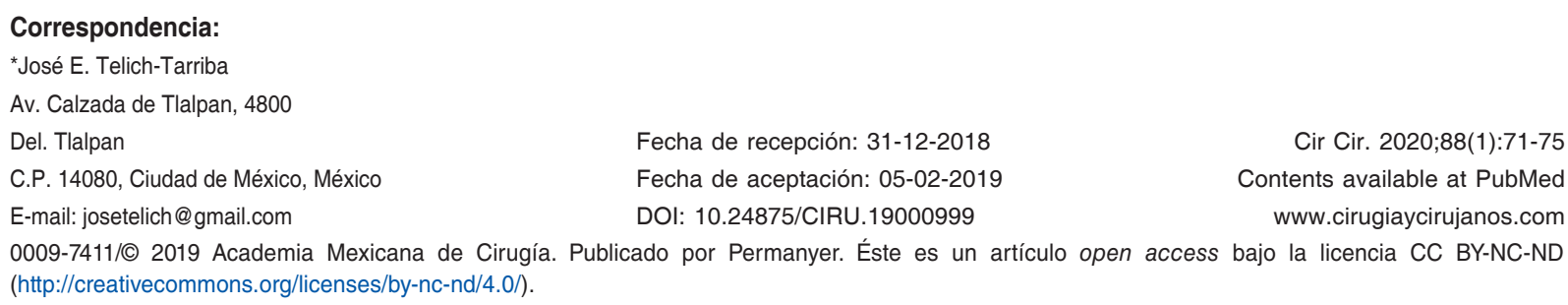

Fecha de recepción: 31-12-2018

Fecha de aceptación: 05-02-2019

C.P. 14080 , Ciudad de México, México

E-mail: josetelich@gmail.com

DOI: 10.24875/CIRU.19000999

0009-7411/@ 2019 Academia Mexicana de Cirugía. Publicado por Permanyer. Éste es un artículo open access bajo la licencia CC BY-NC-ND (http://creativecommons.org/licenses/by-nc-nd/4.0/). 
were included, mean age $11.79 \pm 9.39$ years. The facial nerve showed complete unilateral recruitment in 4 patients, partial bilaterally in 11, 7 showed no activity bilaterally and two had unilateral inactivity. The masseterine was normal in 14 patients, had partial recruitment bilaterally in 4 and unilaterally in 4 cases. The accessory nerve was normal in 20 patients, showed partial recruitment bilaterally in 3 and unilaterally in 1 patients. The hypoglossal was normal in 22 patients, and altered in 2 cases. Conclusion: Patients with Moebius syndrome show several degrees of alteration in electromyographic evaluation of the facial nerve. Electromyography is a useful tool in evaluating potential motor donor nerves for facial reanimation surgery.

Key Words: Electromyography. Facial nerve. Neurophysiology. Facial paralysis. Moebius syndrome.

\section{Introducción}

El síndrome de Moebius es una enfermedad poco común, de etiología desconocida, caracterizada por parálisis bilateral congénita de los nervios facial y abducens. En la literatura internacional se han reportado aproximadamente 300 casos y se calcula que tiene una incidencia de 1 caso por cada 50,000 recién nacidos vivos ${ }^{1}$. Nuestro grupo de investigación publicó la serie de pacientes más grande hasta el momento, incluyendo 115 tratados a lo largo de 5 años ${ }^{2}$.

Además de la presencia de parálisis del sexto y séptimo pares, otros nervios craneales pueden encontrarse paralizados, siendo los más afectados en orden decreciente los pares XII, X, IX, III, VIII, V, IV y $\mathrm{XI}$. Las manifestaciones clínicas suelen pasar desapercibidas al momento del nacimiento, por lo que suele diagnosticarse durante el periodo de lactancia al encontrar problemas para la alimentación, como dificultad para succionar o en la deglución, sialorrea y ausencia de movimientos faciales al llorar'.

La etiología del síndrome es desconocida hasta el momento. Las principales teorías corresponden a alteraciones vasculares o genéticas, con potenciales factores tóxicos asociados. La teoría vascular propone que el síndrome es producido por disrupción de la arteria basilar o las trigeminales durante el desarrollo embrionario, generando zonas de isquemia a nivel central, lo que también explicaría el desarrollo de disfunción en otros pares craneales ${ }^{3}$. La teoría genética está basada en reportes de alteraciones cromosómicas en algunos pacientes, como la deleción del cromosoma 13 o una traslocación 1:11; en otros casos se ha reportado una herencia autosómica dominante, recesiva o ligada al cromosoma $X^{4}$. Debido a esta heterogeneidad no se ha podido determinar una causa genética para el padecimiento. Eventos prenatales como exposición a talidomida, misoprostol, cocaína, ergotamina y benzodiacepinas se han propuesto también en la patogenia del síndrome ${ }^{5-7}$.
Existen múltiples malformaciones asociadas, especialmente las del sistema musculoesquelético, como braquidactilia, sindactilia o artrogriposis, así como la presencia de otros síndromes, como el de Poland y el de Klippel-Feil. En el tórax se han reportado defectos ventriculares septales, dextrocardia y transposición de grandes vasos. Las malformaciones orofaciales incluyen paladar hendido, úvula bífida, microstomía, micrognatia e insuficiencia velofaríngea debida a parálisis del IX par'.

El diagnóstico clínico se basa en los hallazgos de la exploración física. De acuerdo con la presentación de las alteraciones faciales, Terzis clasificó a los pacientes en tres grupos: Moebius completo si hay afección de los pares VII y VI craneales bilateralmente, Moebius incompleto si la afección del VII par es unilateral, y Moebius like si hay afección del VII par con otro nervio craneal excluyendo el VI. Hasta el momento no existen escalas validadas que evalúen la gravedad de la parálisis del nervio facial en los pacientes con síndrome de Moebius ${ }^{8}$.

Los estudios electrofisiológicos evalúan en forma objetiva la función del nervio facial y sus músculos diana, lo que permite determinar la gravedad de su compromiso, estimar su pronóstico y guiar el tratamiento ${ }^{9}$. Debido a la rareza de la enfermedad, existen pocos estudios que evalúen la neurofisiología de la musculatura facial en la población con síndrome de Moebius, por lo que la información disponible es limitada y en muchas ocasiones originada de otras poblaciones con parálisis facial. Hasta el momento, únicamente Verzijl, et al. ${ }^{10}$, Cattaneo, et al. ${ }^{11} \mathrm{y}$ Jaradeh, et al..$^{12}$ han reportado resultados electromiográficos de pacientes con síndrome de Moebius, pero los estudios se han enfocado exclusivamente en el impacto neurofisiológico de la enfermedad y no se han valorado los posibles nervios donadores con fines de planificación preoperatoria.

El objetivo de este trabajo es describir la actividad electromiográfica de la musculatura facial y los principales nervios donadores para reconstrucción facial dinámica en pacientes con síndrome de Moebius atendidos en nuestro servicio. 


\section{Método}

Estudio retrospectivo, observacional y transversal en el que se incluyeron pacientes con síndrome de Moebius atendidos en una nuestra institución entre 2010 y 2016. El síndrome se definió como la presencia de parálisis facial no progresiva e incapacidad para la abducción ocular, presentes desde el nacimiento. Los pacientes fueron divididos en tres grupos de acuerdo con la clasificación de Terzis y Noah:

- Moebius clásico: parálisis facial y de abducens bilateral.

- Moebius incompleto: parálisis bilateral del abducens y parálisis facial con movimiento residual unilateral.

- Moebius like: parálisis facial unilateral acompañada por parálisis de algún otro nervio craneal.

Únicamente se evaluaron pacientes que contaran con electromiografías de musculatura inervada por los nervios facial, masetero, hipogloso y accesorio. Las electromiografías fueron realizadas con electrodos de aguja bipolares colocados en los músculos a evaluar. Los puntos de registro se colocan en el músculo frontal, en el orbicular de los ojos a la altura del canto lateral, en los cigomáticos, el orbicular de la boca y el mentoniano, puntos que corresponden con los ramos temporal, cigomático y mandibular del nervio facial9,13. Para la valoración de los nervios donadores se colocaron electrodos sobre los maseteros para evaluar el ramo maseterino del trigémino, en el trapecio para evaluar el espinal o en la lengua para evaluar la función del hipogloso9,13.

Durante la actividad muscular se determina el porcentaje de unidades motoras reclutadas en comparación con el lado sano o el estándar ${ }^{13}$, por lo que se decidió agrupar los resultados en tres categorías: reclutamiento completo, parcial o nulo.

La información fue recolectada en una base de datos en Microsoft Excel, incluyendo edad, sexo, tipo de Moebius según la clasificación de Terzis y $\mathrm{Noah}^{8}$, y actividad muscular en la electromiografía para cada grupo muscular en ambos lados del cuerpo.

El análisis estadístico se realizó utilizando estadística descriptiva de las características demográficas de los pacientes; las variables continuas se presentan como medidas de tendencia central, y las categóricas como porcentajes.

\section{Resultados}

Durante el periodo de estudio se atendieron 115 pacientes con síndrome de Moebius en la clínica.
Tabla 1. Características demográficas de la muestra

\begin{tabular}{lcc}
\hline & $\mathbf{n}$ & $\%$ \\
\hline $\begin{array}{lcc}\text { Sexo } \\
\text { Hombre }\end{array}$ & 10 & \\
Mujer & 14 & 41.6 \\
& Media & DE \\
\hline Edad & 11.79 & 9.39 \\
\hline & $\mathbf{n}$ & $\%$ \\
\hline Clasificación & & \\
Moebius clásico & 15 & 62 \\
Moebius incompleto & 9 & 37 \\
Moebius like & 0 & 0 \\
\hline DE: desviación estándar. & &
\end{tabular}

Para el presente estudio se incluyeron 24 (14 mujeres y 10 hombres) que contaban con electromiografías de los grupos musculares de interés; la media de edad fue de 11.79 años (desviación estándar: \pm 9.39) (Tabla 1). Quince pacientes (62\%) se clasificaron como Moebius clásico y nueve $(37 \%)$ como Moebius incompleto.

La musculatura del nervio facial presentó reclutamiento unilateral completo en cuatro pacientes $(16.6 \%)$ y parcial bilateral en $12(50 \%)$; en cinco pacientes (20.8\%) hubo ausencia de reclutamiento bilateral, e inactividad unilateral en tres (12.5\%). Los maseteros tuvieron reclutamiento completo bilateral en 14 pacientes $(58.3 \%)$, parcial bilateral en seis $(25 \%)$ y parcial unilateral en cuatro (16.6\%).

El músculo trapecio mostró reclutamiento completo bilateral en 20 pacientes (83.3\%), parcial bilateral en tres $(12.5 \%)$ y parcial unilateral en uno $(4 \%)$. El músculo lingual tuvo actividad normal bilateral en 22 pacientes $(91.6 \%)$, parcial bilateral en uno $(4 \%)$ y parcial unilateral en otro (4\%) (Tabla 2).

Al analizar los resultados según el tipo de síndrome que presentaron los pacientes se observó que en los 15 con síndrome de Moebius completo existió reclutamiento parcial bilateral en la musculatura facial en nueve, ausencia total de reclutamiento en cuatro, reclutamiento parcial unilateral en uno y reclutamiento completo unilateral en uno. En los nueve pacientes con síndrome de Moebius incompleto existió reclutamiento parcial bilateral en tres, reclutamiento parcial unilateral en dos, completo unilateral en tres y ausencia de reclutamiento en uno.

\section{Discusión}

El síndrome de Moebius engloba un conjunto de malformaciones y disfunciones clínicas que ameritan 
Cirugía y Cirujanos. 2020;88(1)

Tabla 2. Actividad electromiográfica de los grupos musculares evaluados

\begin{tabular}{lcccccc}
\hline Grupo muscular & Completo bilateral & Completo unilateral & Parcial bilateral & Parcial unilateral & Nulo bilateral & Nulo unilateral \\
\hline Facial (VII) & - & $4(16.6 \%)$ & $12(50 \%)$ & - & $5(20.8 \%)$ & $3(12.5 \%)$ \\
Maseteros $(\mathrm{V})$ & $14(58.3 \%)$ & - & $6(25 \%)$ & $4(16.6 \%)$ & - & - \\
Trapecio (XI) & $20(83.3 \%)$ & - & $3(12.5 \%)$ & $1(4 \%)$ & - & - \\
Lingual (XII) & $22(91.6 \%)$ & - & $1(4 \%)$ & $1(4 \%)$ & - & - \\
\hline
\end{tabular}

manejo multidisciplinario. Descrito por primera vez en 1880 por von Graefe y en 1888 por Paul Möbius, actualmente se define como la combinación de parálisis facial y del abducens al momento del nacimiento'. La corrección de la función muscular facial es importante, ya que además de restaurar la sonrisa social permite mejorar problemas de lenguaje, la incompetencia oral, el crecimiento del esqueleto facial y la oclusión dentaria ${ }^{14,15}$.

Todo paciente requiere un protocolo de evaluación multidisciplinario integrado por especialistas en pediatría, oftalmología, ortodoncia, otorrinolaringología, rehabilitación, genética médica y ortopedia. El procedimiento de elección hoy en día es la transferencia microquirúrgica de músculo gracilis, pero al no existir función del nervio facial deben emplearse nervios donadores extrafaciales para neurotizar el gracilis, siendo el maseterino, el accesorio espinal y el hipogloso los más utilizados. En nuestro servicio, el nervio maseterino es el de primera elección, seguido por el espinal, mientras que el hipogloso se reserva para casos de parálisis de los anteriores, debido a que su uso puede relacionarse con dificultades para el habla y la deglución ${ }^{14}$.

La elección del nervio donador dependerá de una exploración clínica exhaustiva, complementada por los resultados de una evaluación electromiográfica. La literatura relacionada con los aspectos neurofisiológicos del síndrome de Moebius es limitada; hasta el momento únicamente se han publicado tres estudios sobre el tema. El estudio más antiguo es el de Jaradeh, et al..$^{12}$, quienes encontraron una reducción en la actividad muscular facial de siete pacientes. En 2005, Verzijl, et al. ${ }^{10}$ reportaron los resultados de seis pacientes, encontrando ausencia de actividad de la musculatura facial bilateral en tres $(50 \%)$ y unilateral en los otros tres (50\%); sin embargo, en estos últimos solo se evaluó una hemicara. Cattaneo, et al. ${ }^{11}$, en 2006, evaluaron 17 pacientes con electromiografía y reportaron tres (17.6\%) sin respuestas bilaterales, cuatro $(23.5 \%)$ con actividad normal bilateral y diez
(58.8\%) con actividad variable dependiendo del músculo estudiado; además, valoraron la actividad de los maseteros y encontraron función normal en el $94 \%$ de los pacientes.

Comparado con estudios previos, nuestro trabajo muestra hallazgos interesantes. Se encontraron resultados normales en menos del $20 \%$ de los pacientes, comparable con los reportes previos, así como variaciones en la actividad muscular que van desde reducciones parciales hasta la ausencia de respuesta en prácticamente el $80 \%$ de los casos, lo cual es ligeramente mayor que en estudios anteriores. Destaca en nuestro estudio que se trata del primero que evalúa en forma integral los nervios donadores más utilizados en reconstrucciones dinámicas microquirúrgicas y que reporta el estado de los músculos trapecio y lingual, cuyos resultados electromiográficos corresponden a los grados de afección observados en otras series clínicas. Llama la atención que en la serie de Verzijl, et al..$^{10}$ los maseteros mostraron actividad normal en el $94 \%$ de los casos, mientras que en nuestra serie esto solo ocurrió en el $58 \%$.

El presente estudio tiene múltiples fortalezas: es el primero en evaluar en forma integral los resultados de electromiografía de la musculatura facial y de los nervios donadores empleados en reconstrucción dinámica, así como el de mayor tamaño de muestra hasta la fecha. Dentro de sus desventajas se encuentra la naturaleza retrospectiva del estudio, así como limitar el reporte de los resultados a tres categorías globales para toda la cara, pues es sabido que el tercio inferior generalmente presenta actividad residual, lo que podría afectar las evaluaciones finales.

\section{Conclusiones}

La electromiografía es un recurso útil en la evaluación preoperatoria de los pacientes con síndrome de Moebius, ya que permite evaluar objetivamente la función de los músculos de la expresión facial y los 
nervios candidatos a donadores para los procedimientos de reanimación dinámica.

\section{Financiamiento}

El presente trabajo se realizó con apoyo de la Beca Carlos Slim en Salud.

\section{Conflicto de intereses}

Los autores declaran que no existe conflicto de intereses.

\section{Responsabilidades éticas}

Protección de personas y animales. Los autores declaran que para esta investigación no se han realizado experimentos en seres humanos ni en animales.

Confidencialidad de los datos. Los autores declaran que han seguido los protocolos de su centro de trabajo sobre la publicación de datos de pacientes.

Derecho a la privacidad y consentimiento informado. Los autores han obtenido el consentimiento informado de los pacientes y/o sujetos referidos en el artículo. Este documento obra en poder del autor de correspondencia.

\section{Bibliografía}

1. Singham J, Manktelow R, Zuker RM. Möbius syndrome. Semin Plast Surg. 2004;18:39-45.

2. Arrieta-Joffe P, Pérez-Dosal M, Ortiz-de-Zárate G, Cardenas-Mejia A. Estudio clínico, citogenético, molecular y de imagen de los pacientes con síndrome de Moebius del Hospital General "Dr. Manuel Gea González", Ciudad de México. Cir Plast Iberolatinoam. 2017;43:395-400.

3. Bavinck JN, Weaver DD. Subclavian artery supply disruption sequence: hypothesis of a vascular etiology for Poland, Klippel-Feil and Möbius anomalies. Am J Med Genet. 1986;23:903-18.

4. Verzijl HT, van der Zwaag B, Cruysberg JR, Padberg GW. Möbius syndrome redefined: a syndrome of rhombencephalic maldevelopment. Neurology. 2003;61:327-33.

5. Shepard TH. Möbius syndrome after misoprostol: a possible teratogenic mechanism. Lancet. 1995:346:780.

6. Graf WD, Shepard TH. Uterine contraction in the development of Möbius syndrome. J Child Neurol. 1997;12:225-7.

7. Courtens W, Vamos E, Hainaut M, Vergauwen P. Moebius syndrome in an infant exposed to in utero benzodiazepines. J Pediatr. 1992;121:833-4.

8. Terzis JK, Noah EM. Möbius and Möbius-like patients: etiology, diagnosis and treatment options. Clin Plast Surg. 2002;29:497-514.

9. Wink JD, Goldstein JA, Paliga JT, Taylor JA, Bartlett SP. The mandibular deformity in hemifacial microsomia: a reassessment of the Pruzansky and Kaban classification. Plast Reconstr Surg. 2014;133:174e-81e.

10. Verzijl HT, Padberg GW, Zwarts MJ. The spectrum of Mobius syndrome: an electrophysiological study. Brain. 2005;128:1728-36.

11. Cattaneo L, Chierici E, Bianchi B, Sesenna E, Pavesi G. The localization of facial motor impairment in sporadic Möbius syndrome. Neurology. 2006;66:1907-12.

12. Jaradeh S, D'Cruz O, Howard JF Jr, Haberkamp TJ, Konkol RJ. Mobius syndrome: electrophysiologic studies in seven cases. Muscle Nerve. 1996;19:1148-53.

13. Fattah A, Gurusinghe A. Facial nerve grading instruments: systematic review of the literature and suggestion for uniformity. Plast Reconstr Surg. 2015;135:569.

14. Cardenas-Mejia A, Palafox D. Facial reanimation surgery in Möbius syndrome: experience from 76 cases from a tertiary referral hospital in Latin America. Ann Chir Plast Esthet. 2018;63:338-42.

15. Palafox D, Arrieta-Joffe P, Cardenas Mejia A. Tratamiento quirúrgico reconstructivo actual del síndrome de Moebius. Cir Plast. 2014;24: 136-44. 